
\title{
25 Research Square \\ The Development of Task Sharing Policy and Guidelines in Kenya
}

\section{Rosemary Kinuthia ( $\square$ rkinuth@emory.edu )}

Emory University Nell Hodgson Woodruff School of Nursing https://orcid.org/0000-0003-1230-221X

\section{Andre Verani}

Centers for Disease Control and Prevention

\section{Jessica Gross}

Centers for Disease Control and Prevention

\section{Rose Kiriinya}

Emory University Kenya Health Workforce Project

\section{Kenneth Hepburn}

Emory University Nell Hodgson Woodruff School of Nursing

\section{Jackson Kioko}

Kenya Ministry of Health

\section{Agnes Langat}

Centers for Disease Control and Prevention

\section{Abraham Katana}

Centers for Disease Control and Prevention

\section{Agnes Waudo}

Emory University Kenya Health Workforce Project

\section{Martha Rogers}

Emory University Nell Hodgson Woodruff School of Nursing

\section{Research Article}

Keywords: Task sharing, Task shifting, Human resources for health, Health workforce shortage, Universal healthcare, Kenya, sub-Saharan Africa

Posted Date: December 20th, 2021

DOI: https://doi.org/10.21203/rs.3.rs-1156828/v1

License: (9) (1) This work is licensed under a Creative Commons Attribution 4.0 International License. Read Full License 


\section{Abstract}

\section{Background:}

The global critical shortage of health workers prevents expansion of healthcare services and universal health coverage. Like most countries in sub-Saharan Africa, Kenya's healthcare workforce density of 13.8 health workers per 10,000 population falls below the World Health Organization (WHO) recommendation of at least 44.5 doctors, nurses, and midwives per 10,000 population. In response to the health worker shortage, the WHO recommends task sharing, which is a strategy to alleviate the burden on certain health workers through the redistribution of tasks. To improve the utilization of human and financial health resources in Kenya for HIV and other essential health services, the Kenya Ministry of Health (MOH) in collaboration with various institutions developed national task sharing policy and guidelines (TSP). To advance task sharing, this article describes the process of developing the Kenya TSP.

\section{Methods:}

The development and approval of Kenya's TSP occurred from February 2015 to May 2017. The U.S. Centers for Disease Control and Prevention (CDC) allocated funding to Emory University through the United States President's Emergency Plan for AIDS Relief (PEPFAR) Advancing Children's Treatment initiative. After obtaining support from leadership in Kenya's $\mathrm{MOH}$ and health professional institutions, the TSP team conducted a desk review of policies, guidelines, scopes of practice, task analyses, grey literature, and peer-reviewed research. Subsequently, a policy advisory committee was established to guide the process and worked collaboratively to form technical working groups to draft the policy.

\section{Results:}

The collaborative, multidisciplinary process led to the identification of gaps in service delivery resulting from health workforce shortages. This facilitated the development of the Kenya TSP, which provides a general orientation of task sharing in Kenya. The guidelines list priority tasks for sharing by various cadres as informed by evidence, such as HIV testing and counseling tasks. The TSP documents were disseminated to all county healthcare facilities in Kenya.

\section{Conclusions:}

Task sharing could increase access to healthcare services in resource-limited settings. To advance task sharing, TSP and clinical practice could be harmonized, and necessary adjustments made to other policies that regulate practice. Revisions to pre-service training curricula could be conducted to ensure health professionals have the requisite competencies to perform shared tasks. Monitoring and evaluation could ensure that task sharing is implemented appropriately to ensure quality outcomes.

\section{Background}


Human resources for health $(\mathrm{HRH})$ is an essential health system pillar according to the World Health Organization (WHO) Health System Building blocks, and the WHO advises health systems have adequate $\mathrm{HRH}$ engaged in service delivery to improve population health (1). To attain the Sustainable Development Goals (SDGs), the WHO recommends a health workforce density of 44.5 doctors, nurses and midwives per 10,000 population (2). However, there is a chronic shortage of health workers globally; the Global Strategy on Human Resources for Health: Workforce 2030 reports an estimated global needs-based shortage of over 17 million health professionals, including over 9 million nurses and midwives and 2.6 million physicians, and the remainder includes all other health worker cadres. The greatest shortages are in regions with the highest unmet health needs, such as South East Asia and sub-Saharan Africa (2).

Nurses and midwives represent the largest group of health professionals and account for approximately half of the global HRH shortage (3). According to WHO estimates, the global nursing and midwifery workforce shortage will reduce from 9 million to 7.6 million by 2030 (2). While the global nursing workforce is expected to grow, the nursing and midwifery shortage in Africa is estimated to worsen from 1.8 million in 2013 to 2.8 million in 2030 (4). This outcome will limit access to healthcare services in the regions most adversely affected, thus, prompting health systems in LMICs to focus on alternative strategies.

The chronic HRH shortage challenging health systems makes it difficult to provide universal HIV services, attain the SDGs, and enhance population health. The global burden of disease is increasing as populations are living longer (5). Additionally, low and middle income countries (LMICs) are facing the double-burden of infectious diseases and rising prevalence of non-communicable diseases, leading to an increased demand for access and provision of health services (6). The existing HRH in LMICs, however, is not adequate to meet this increasing demand.

Kenya is not exempt from the global HRH crisis; while there are efforts in place to scale up the national health workforce, the number of active healthcare workers remains far below the current WHO recommendations. As reported in the Kenya Health Workforce Report, there is a total of 53,118 active doctors, clinical officers, and nurses and midwives; thus, the health worker to population ratio in the country is 13.8 providers per 10,000 individuals in the nation's population (7). This ratio is less than one third the WHO critical threshold recommendation of 44.5 providers per 10,000 individuals. Similar to most LMICs, Kenya's health workforce is largely comprised of nursing professionals who provide a majority of healthcare services to the population. The nurses, however, are overburdened as there are only 8.3 nurses per 10,000 population compared to WHO's recommendation of 25 nurses per 10,000 population (7). Given Kenya's critical shortage of health workers, optimization of task sharing with its health workers cadres (Appendix B) may promote efficient delivery of health services.

Kenya's nursing workforce is affected by a variety of factors. A significant contributor to the depletion of Kenya's nursing workforce is outmigration with an estimated $6 \%$ of the nursing workforce applying to outmigrate (8). Another growing concern is the rising rate of pre-service attrition between enrollment in a training institution and registration with the nursing council. Pre-service attrition of Kenyan nurses 
doubled from $4.3 \%$ in 1999 to $8.2 \%$ in 2004 . This is due in part to onerous faculty workloads and insufficient clinical mentorship for students (9) .

Kenya has made strategic investments to scale up the nursing workforce, including increasing the national capacity to train nursing professionals by focusing on the expansion of nurse training institutions. This approach led to a 32.5\% increase in the number of nursing schools, from 77 to 102 between 2006 and 2015 (7). This expansion subsequently led to increased student enrollment into nursing programs, either as new entrants or advanced practice (7). Additionally, the United States President's Emergency Plan for AIDS Relief (PEPFAR) has supported training for thousands of health workers (10). Despite these investments, the healthcare workforce shortage has remained persistent.

While increasing the number of nurses has contributed to addressing the HRH shortage in Kenya, it is still not adequate to meet the needs of the health system. Like most of sub-Saharan Africa, Kenya has a large number of persons living with HIV, which has strained the healthcare system and its workforce. In 2016, there were 1.6 million people living with HIV, 62,000 new infections, and 36,000 AIDS-related deaths (11). Kenya's HIV prevalence rate is $5.9 \%$; however, some regions have disproportionally higher rates than the rest of the nation. For example, Homa Bay, a county in rural Western Kenya, has a HIV prevalence rate of $26 \%$, which is almost 4.5 times the national HIV prevalence rate $(12,13)$. The HIV rates in Kenya's Western region are comparable to eSwatini (formerly known as Swaziland), which is known to have the highest rates of HIV in the world at $27.4 \%$ (14). The model of physician run clinics, common in High Income Countries, is not feasible for Kenya and other LMICs, given the scarcity of physicians, and health systems have sought other approaches to delivering HIV care, treatment for other illnesses, as well as health promotion and disease prevention services (15).

In light of the global disease burden, HIV pandemic, and critical shortage of trained health workers, the WHO released guidelines on task shifting as one approach to address HRH concerns and increase access to HIV care and other health services. According to WHO, task shifting is "a process whereby specific tasks are moved, where appropriate, to health workers with shorter training and fewer qualifications"(16). If implemented appropriately, task shifting is intended to improve health care coverage by utilizing more widely available cadres, such as nurses and clinical officers, to improve the efficiency of already existing $\mathrm{HRH}$. When the WHO report was released in 2007, task shifting was a recommended solution to reaching the difficult to attain Millennium Development Goals for health and to achieve universal access to HIV services. Over time the terminology evolved to task sharing and was formally introduced in the scientific literature by the Institute of Medicine (IOM) in 2010. The IOM introduced the concept of task sharing as a strategy for capacity building, prevention, treatment, and care of HIV/AIDS in Africa. Task sharing addresses bottlenecks in the delivery of health services through efficient use of existing HRH, whereby "physicians, nurses, dentists, and other health professionals delegate health care responsibilities and relevant knowledge to others, including community health workers" (17). In addition to encouraging collaboration, the IOM recommended that task sharing focus on the clear delegation of roles and promotion of competency-based training for health workers taking on new tasks. 
Task shifting and task sharing are used in a variety of public health settings to meet the demand for health services and address workforce shortages. Although WHO recommended formal task shifting in the context of addressing the HIV epidemic, its application was extended to address other areas such as maternal and newborn health care (18) using WHO's maternal and newborn task shifting guidelines (19). Other areas where task shifting has been implemented include reproductive health (20) and tuberculosis care (21). The use of task sharing as a model of delivering care is gaining popularity in under-resourced regions (21). However, implementation has often been informal and established organically to adapt to HRH shortages (22). Task sharing is used widely in sub-Saharan Africa, increasing access to healthcare services, and yielding positive health outcomes $(23,24)$. However, despite the benefits of task sharing, and its informal but widespread use in Africa, there is a scarcity of evidence documenting the process of developing and implementing task sharing guidelines. Guidelines and policies are needed to facilitate the process of task distribution to alleviate the workforce burden among health workers in an organized and systematic manner.

In 2015, Emory University, in collaboration with the Kenya Ministry of Health (MOH), the U.S. Centers for Disease Control and Prevention (CDC), and PEPFAR sought to advance task sharing to promote equitable access to universal health coverage (UHC), including HIV services at the national, county, sub-county and community levels in Kenya. Early in this process, the $\mathrm{MOH}$ partnered with several institutions to establish the initial advisory committee overseeing the development of the 2017-2030 Kenyan Task Sharing Policy and Guidelines (TSP) (25). The goals of the TSP were to improve the utilization of human and financial health resources by: (i) Establishing a comprehensive national framework for task sharing, (ii) Equipping health workers with relevant knowledge, skills, and competencies, (iii) Ensuring the delivery of quality health services by all health workers and (iv) Allocating resources to sustain task sharing implementation, monitoring and evaluation. As stated in the Kenya Task Sharing Policy, it is intended to "facilitate enhanced quality service delivery in Kenya through the implementation of an integrated task sharing framework, improving access to essential health services, including HIV/AIDS prevention, care and treatment" $(20,26)$. This article describes the development process of the Kenya TSP.

\section{Methods}

\section{Phase 1 (February 2015- September 2015)}

CDC allocated funding through the PEPFAR Advancing Children's Treatment initiative to facilitate development of Kenya's TSP. The lack of advanced practitioners trained and authorized to provide HIV treatment to children was identified as a barrier to PEPFAR's HIV treatment coverage for children in Kenya. In February 2015, CDC Kenya and Kenya's health professional leaders began to conceptualize the initial ideas to address HRH challenges in the country. In August 2015, the Emory University Kenya Health Workforce Project met with the Kenyan Director of Medical Services (DMS) to obtain high-level buy-in to develop the TSP guidelines and policy for advancement of formal task sharing in Kenya to address HRH needs. The Kenya $\mathrm{MOH}$ identified key members for the establishment of a Policy Advisory Committee (PAC) to steer the initiative. The initial PAC stakeholders consisted of the Kenya MOH, county 
representatives, training institutions, regulatory and professional bodies, faith-based organizations, and partner agencies such as WHO, United States Agency for International Development (USAID), and CDC.

\section{Phase 2 (September 2015-October 2015)}

In September 2015, the PAC, which included the Kenya MOH and a wide range of institutions and professionals such as nurses and physicians, held its first meeting to identify the best approach for the development of the task sharing guidelines and policy (see Appendix A for a comprehensive list of all members of the PAC). The stakeholders worked collaboratively to conduct a stakeholder analysis, as well as to identify the scope of the task sharing policy, the best approach for policy development, and the level of approval needed in the Kenya $\mathrm{MOH}$. To guide the process, the PAC conducted a desk review (27) of task sharing policies in other countries, scopes of practice for various healthcare cadres, task analyses of each cadre, grey literature, and peer-reviewed research. The process identified gaps in the existing models used to deliver healthcare in Kenya and identify opportunities to utilize the competency and skills of various cadres more effectively through task sharing. The PAC decided to develop a task sharing policy inclusive of HIV care and other health services essential for UHC. This initial decision led to a lengthier, more complex process than an HIV specific task sharing policy with the added benefit of resulting in a policy document highly relevant to Kenya's goal to achieve UHC, as well as PEPFAR's goal to expand HIV services.

The PAC subsequently established five technical working groups (TWGs) to advance the development of the Kenyan TSP within their respective thematic areas. To emphasize the importance of the TSP, the Kenya Principal Secretary for Health officially launched the TWGs in September 2015. The PAC recommended areas of concentration for the TWGs informed by the desk review. The first TWG: Introduction and Evidence, conducted a mapping exercise and focused on reviewing evidence and background information regarding task sharing policy in Kenya's health system. This group worked together to provide the aim and objectives of the TSP and to conduct a situational analysis. The second TWG: Legal and Regulatory, researched and analyzed existing laws, regulations, and policies pertinent to task sharing and recommended harmonization of national laws, policies, regulations, and guidelines in support of task sharing. The third TWG: Training, focused on identifying requirements to equip health workers with the necessary knowledge, skills, and competencies to provide essential healthcare services. Stakeholders conducted a task analysis of each cadre, identifying their training needs, then collaborating to explore best practices to promote quality healthcare through pre-service, internship, in-service training, and continuing professional development (CPD). The fourth TWG: Service Delivery, worked together to identify key service areas and ensure the service delivery guidelines were comprehensive for all six levels of Kenya's healthcare system. The fifth TWG: Implementation, Monitoring and Evaluation applied evidence-based principles of monitoring and evaluation to develop an implementation checklist, identify data collection methodologies, and develop indicators to monitor the progress of TSP implementation.

\section{Phase 3 (October 2015-October 2016)}


The TWGs convened periodically over the course of a year to develop the task sharing policy and guidelines. The groups utilized an iterative process involving three collaborative rounds of in-person meetings and online communication to develop the policy. Round 1 of the TWG meetings took place from October 27th to November 4th, 2015. During this time, the groups developed a draft outline for the policy. Round 2 of the TWG meetings, which took place from December 1st to 9th, 2015, resulted in the development of an initial draft of the TSP policy, with each TWG drafting its respective section. Revision of the initial draft took place from February 2nd to 9th, 2016 during round 3 of the TWG meetings. For about three months after the round 3 meetings, the team collaborated to complete several activities that led to finalizing the policy. These activities included: finalizing the comprehensive TSP guidelines, reviewing of the guidelines with healthcare workers $(\mathrm{HCW})$ who were members of the PAC, revision of policy using feedback from HCWs and convening of the PAC to finalize the policy. PAC members reviewed the TSP to ensure integration and coherence, since the policy was drafted section-by-section by the respective TWGs. The final TSP policy and guidelines were submitted to the Kenya $\mathrm{MOH}$ on May 15th, 2016.

\section{Phase 4 (April-May 2017)}

After submission of the final documents to the $\mathrm{MOH}$, the Cabinet Secretary and DMS reviewed and approved the TSP to become government policy. To further emphasize the importance of the guidelines, an official launch of the policy took place during a ceremony involving the MOH's DMS, CDC officials, county health officials, health professional regulatory board representatives, and other stakeholders. Thereafter, the policy and guidelines were disseminated to all county representatives.

\section{Phase 5 (Ongoing)}

The final phase of the process is implementation, monitoring, and evaluation. Activities to facilitate this final phase are ongoing and Emory University in collaboration with the $\mathrm{MOH}$ is currently focusing on wide dissemination of the TSP and guidelines. TSP sensitization to increase awareness of the policy and guidelines has already taken place in 10 select counties, and there are plans to sensitize and train all 47 counties in Kenya.

\section{Results}

The TSP process resulted in the development of the Kenya 2017-2030 Task Sharing Policy and Guidelines (25). The policy document provides a brief general orientation to task sharing and key aims of task sharing policy. The guidelines list priority health-related task sharing activities by cadre with the evidence base. The guidelines are organized into six chapters with topics ranging from introduction to recommendations for the future. Chapter 1: Introduction presents background information on Kenya's health indicators, overview of the health system, and the health worker shortage crisis; thus, making a case for why introducing task sharing in Kenya would be beneficial to addressing the workforce shortages. The chapter also discusses the global evidence-based recommendations for task sharing from the WHO. Chapter 2: Legal, Regulatory and Policy Framework highlights various Kenyan laws, regulations 
and policies, and reviews whether they enable or restrict the implementation of task sharing in the country while urging harmonization of Kenya's laws, regulations, and other policies in support of task sharing. Chapter 3: Training and Education presents an overview of health training in Kenya and summarizes the training provided to select cadres and addresses the need for specialized training and continuing education for these cadres to facilitate implementation of task sharing. Chapter 4: Task Sharing by Cadre and Level presents targeted cadres and a large number and variety of tasks that may be shared by each cadre after ensuring competency to perform the task. See Appendix B for a list of cadres targeted for task sharing. To facilitate implementation, the information is presented in tables that are easy to read and interpret. These tables constitute approximately half of the entire document, as several hundred tasks are listed alongside the evidence base for sharing each task with each cadre. These tasks include HIV rapid testing, HIV treatment, HIV Pre-Exposure Prophylaxis, medical male circumcision, TB case identification, malaria rapid diagnostic testing, micronutrient supplementation, and provision of immunizations, among many others. See Appendix C for an example of the HIV testing and counseling table included in the TSP document. Chapter 5: Monitoring and Evaluation (M\&E) briefly describes the areas that M\&E of the TSP will cover as well as the guiding principles and M\&E framework of the TSP. Chapter 6: Recommendations is the final chapter and makes suggestions for the way forward, centering around five key areas: i) adoption and implementation; ii) harmonization of laws, policies and regulations; iii) training; iv) service delivery; and v) monitoring and evaluation of TSP implementation.

\section{Discussion}

This initiative highlighted several areas where task sharing may be used effectively to advance the health of the Kenyan population and attain UHC. The process of developing the TSP guidelines and policy was successful and encouraged collaboration between the stakeholders. It is a critical step towards providing quality health services and presents opportunities for the implementation of the TSP guidelines and policy to optimize delivery of key health interventions. The documents were disseminated electronically to all counties in Kenya; however, to complement the guidelines and policy there are several pending items to be addressed to advance task sharing implementation. Revision of certain legislation in Kenya may be considered to lift restrictive laws prohibiting the sharing of certain tasks. For example, as noted in the guidelines document, the Public Health Act restricts disease reporting to medical officers even though many health facilities in Kenya lack even one medical officer. Further, The Clinical Officers Act does not authorize HIV treatment by private sector clinical officers. As Kenya moves towards UHC, private sector providers will prove essential.

Revision of scopes of practice and schemes of service for the various cadres delivering healthcare services is recommended. As noted above, there are tasks certain health professionals can perform competently; however, the tasks may not be covered by the cadre's scope of practice, as defined by the regulatory agency. Regulatory authorities could, therefore, work collaboratively to address how all cadres can practice to the full scope of their training. In some cases, cadres in under-resourced areas are practicing beyond their official scope of practice because of clients' needs; however, there may be the risk of liability for performing certain tasks, even if performed competently. Nurses have expressed frustration 
regarding only being allowed to prescribe from a limited range of medications despite expertise and training, thus, unnecessarily limiting their scope of practice (26). For example, while Kenyan nurses technically cannot prescribe therapy such as intravenous fluids, in rural areas where it is likely that the only provider available is a nurse, the nursing professional would have to prescribe and administer fluids when necessary, despite current nursing regulation. Revising scopes of practice and schemes of service will allow such health workers to practice confidently without fear of professional discipline or legal action.

Capacity development of stakeholders is key for successful implementation (28). Thus, implementation of the TSP policy and guidelines will benefit from capacity building to ensure all health workers involved in the delivery of health services have adequate knowledge, skills, and competencies to perform shared tasks. Ideally, capacity building would take place across public and private sector workplaces,

professional association forums, and training institutions. The $\mathrm{MOH}$ could explore the benefits of revising and updating the schemes of service to support task sharing. Employers are highly encouraged to ensure new tasks are authorized and included in job descriptions. For already credentialed health workers, training institutions could consider incororating CPD and continuing medical education (CME) to increase knowledge, skills, and competencies to facilitate task sharing. To meet the needs of the future healthcare workforce, the TSP encourages training institutions to revise the existing training curricula at all levels to include new tasks supportive of task sharing. Additionally, regulatory boards and councils could consider revising the curriculum for certain cadres as part of the accreditation process (initial or renewal) for training institutions.

\section{Challenges/barriers:}

In Kenya, an initial barrier to the TSP development process was obtaining high-level buy-in to move forward with the process. The TSP policy and guidelines development demanded time, resources, and dedication of key individuals from the $\mathrm{MOH}$. The $\mathrm{MOH}$ is faced by many competing priorities, some of which had potential to be impacted by TSP process. However, adequate preparation by parties interested in furthering task sharing prior to the meeting with the $\mathrm{MOH}$ facilitated buy-in. In addition, TSP project champions with prior experience in the $\mathrm{MOH}$ secured key meetings to gain support and advance policy development with $\mathrm{MOH}$ leadership.

As with other collaborative processes, engaging all stakeholders and coordinating the logistics to identify meeting times suitable to all parties is challenging. This was no different for the TSP team. Oftentimes senior officials involved in the TWGs had competing tasks and could not participate in meetings. Some leaders designated their assistants as delegates in their absence. Additionally, involving stakeholders across the health sector was complex, and some institutions were not fully represented. However, the flexibility of the engaged parties facilitated advancement of the TSP development. Meetings included available stakeholders and delegates. Individuals unable to join received meeting briefings.

The process of developing the task sharing policy and guidelines was complex and rigorous, requiring dedication and commitment from all stakeholders. While slow email responses caused some delays, the 
policy development process advanced to meet budgetary and timeline constraints. Another major barrier encountered during the policy development process was a nationwide nurses and doctors strikes that lasted for months. The strikes took attention away from the TSP and some of the TWG meetings had to be delayed because $\mathrm{MOH}$ senior management representatives were dealing with the strike issues. Dissemination of the documents, therefore, mainly occurred via email, and despite wide dissemination, there was no way to assure frontline workers reviewed the documents.

A final challenge is the Kenyan court ruling in April of 2019 in the case brought by the Association of Kenya Medical Laboratory Scientific Officers against the Ministry of Health's TSP (29). In that case, Kenya's High Court stopped implementation of the TSP, at least temporarily.

\section{Lessons learned:}

The TSP development process provided insights to inform task sharing policy work in other countries. Most important for the success of the TSP was the high-level political will from the $\mathrm{MOH}$ and involvement of a wide range of stakeholders from the health sector that ensured ownership and buy-in. Best-practices in research encouraged collaboration among professionals to get a well-rounded perspective. During the collaborative process, stakeholders identified that sharing roles among the TWGs ensured a focused and faster process. To advance collaboration, the TSP team shared progress reports with the human resources for health interagency coordinating committee, whereby the counties are represented in this forum, securing essential county level input. Since counties are responsible for health service delivery under Kenya's Constitution, their involvement and buy-in was and will continue to prove fundamental to the TSP's success.

The TSP PAC engaged the $\mathrm{MOH}$ review team early in the process and received orientation on the flow of government policy, which enhanced the efficiency of the policy development process and enabled the PAC to meet the time constraints discussed above. While technology facilitates remote meetings in many parts of the world, the TWGs found that meeting in person was most productive, and much progress was made during the in-person meetings.

\section{Conclusion}

The process of developing the Kenya TSP demonstrates the value of working collaboratively. The development of a national task sharing policy establishes a framework in Kenya for innovative and differentiated service delivery models for essential health services. The TSP documents will help to advance task sharing in Kenya and address the workforce shortages; however, it is anticipated that implementation in all 47 counties will be challenging. Crucial to this implementation in all counties will be to follow-up on the recommendations regarding revision of legislation, scope of practice, capacity building, schemes of service and curriculum revisions. To evaluate the effectiveness of task sharing, monitoring and evaluation per the guidelines could follow implementation. Evaluation of the TSP guidelines will inform whether the guidelines are helpful in addressing the challenges associated with 
health worker shortages as intended. Additionally, implementation of the TSP guidelines could facilitate successful scale up of UHC in Kenya.

\section{List Of Abbreviations}

$\mathrm{HRH}$ - Human resources for health

WHO- World Health Organization

SDG- Sustainable Development Goals

LMICs- Low- and middle-income countries

IOM - Institute of Medicine

$\mathrm{MOH}-$ Ministry of Health

CDC- Centers for Disease Control and Prevention

UHC- Universal health coverage

TSP- Task sharing policy and guidelines

PEPFAR- President's emergency plan for AIDS relief

DMS- Director of medical services

PAC- Policy advisory committee

USAID- United States Agency for International Development

TWG- Technical working groups

NASCOP- National AIDS and STI Control Programme

CPD-Comprehensive continuing professional development

HCW- Healthcare workers

CME- Continuing medical education

\section{Declarations}

\section{Ethics approval and consent to participate}

Not applicable 


\section{Consent for publication}

All the authors have reviewed the final manuscript and provide consent for publication

\section{Availability of data and materials}

The data that support the development of the Kenya TSP documents are available from the Kenya Ministry of Health. Summaries of the findings were published in the TSP documents, which are publicly available and can be accessed via the following hyperlink: https://www.hesma.or.ke/wpcontent/uploads/2017/02/Task-Sharing-Guideline-2017.pdf

\section{Competing interests}

There are no commercial associations that might pose a conflict of interest in connection with this manuscript.

\section{Funding}

This work was funded under the terms of the cooperative agreement number U360E000002 with funding from the U.S. President's Emergency Plan for AIDS Relief (PEPFAR) through the United States Centers for Disease Control and Prevention, Division of Global HIV \&TB.

\section{Authors' contributions}

RK, AW, JG, and AV contributed to the conception and development of the manuscript. All authors were involved in drafting the manuscript and all the authors contributed by their comments of improvement in several revisions to reach a final manuscript. All authors read and approved the final manuscript.

\section{Acknowledgements}

We would like to acknowledge PEPFAR and CDC for their financial support of the project. We also thank the Kenya Ministry of Health for commissioning this work, members of the policy advisory committee and technical working groups for their participation in the TSP process and working tirelessly to ensure the success of this project.

\section{Disclaimer}

The findings and conclusions in this report are those of the authors and do not necessarily represent the official position of the Centers for Disease Control and Prevention.

\section{References}

1. Organization WH. Monitoring the building blocks of health systems: a handbook of indicators and their measurement strategies. Geneva, Switzerland: World Health Organization; 2010. 
2. World Health Organization. Global strategy on human resources for health: workforce 2030. Geneva, Switzerland; 2016.

3. World Health Organization. Nursing and Midwifery: World Health Organization; 2018 [Available from: http://www.who.int/mediacentre/factsheets/nursing-midwifery/en/.

4. World Health Organization. Global Health Observatory (GHO) data 2018 [Available from: http://www.who.int/gho/health_workforce/nursing_midwifery_density/en/.

5. GBD 2015 Mortality and Causes of Death Collaborators. Global, regional, and national life expectancy, all-cause mortality, and cause-specific mortality for 249 causes of death, 1980-2015: a systematic analysis for the Global Burden of Disease Study 2015. Lancet (London, England). 2016;388(10053):1459-544.

6. Essue BM, Laba M, Knaul F, Chu A, Minh HV, Nguyen TKP, et al. Economic Burden of Chronic III Health and Injuries for Households in Low- and Middle-Income Countries. In: rd, Jamison DT, Gelband H, Horton S, Jha P, Laxminarayan R, et al., editors. Disease Control Priorities: Improving Health and Reducing Poverty. Washington (DC): The International Bank for Reconstruction and Development / The World Bank (c) 2018 International Bank for Reconstruction and Development / The World Bank.; 2017.

7. Kenya Ministry of Health. Kenya Health Workforce Report: The Status of Healthcare Professionals in Kenya, 2015. 2015.

8. Gross JM, Rogers MF, Teplinskiy I, Oywer E, Wambua D, Kamenju A, et al. The impact of outmigration on the nursing workforce in Kenya. Health services research. 2011;46(4):1300-18.

9. Appiagyei AA, Kiriinya RN, Gross JM, Wambua DN, Oywer EO, Kamenju AK, et al. Informing the scaleup of Kenya's nursing workforce: a mixed methods study of factors affecting pre-service training capacity and production. Human resources for health. 2014;12:47.

10. Centers for Disease Control and Prevention. Building public health workforce capacity: Centers for Disease Control and Prevention; 2017 [Available from: https://www.cdc.gov/globalhealth/countries/kenya/what/building.htm.

11. AIDSinfo. 2016 County Factsheets: Kenya [Available from: http://aidsinfo.unaids.org.

12. National AIDS Control Council. Kenya HIV County Profiles 2016. Nairobi, Kenya: National AIDS and STI Contol Programme; 2016.

13. National AIDS control Council. Kenya AIDS resonse progress report Nairobi, Kenya; 2016.

14. Joint United Nations Programme on HIV/AIDS. Country fact sheet: eSwatini UNAIDS; 2017 [Available from: http://www.unaids.org/en/regionscountries/countries/swaziland.

15. McGuire M, Ben Farhat J, Pedrono G, Szumilin E, Heinzelmann A, Chinyumba YN, et al. Task-sharing of HIV care and ART initiation: evaluation of a mixed-care non-physician provider model for ART delivery in rural Malawi. PLoS One. 2013;8(9):e74090.

16. World Health Organization. Task shifting : rational redistribution of tasks among health workforce teams : global recommendations and guidelines. Geneva: World Health Organization; 2007. 
17. Institute of Medicine Committee on Envisioning a Strategy for the Long-Term Burden of HIVAAN, Interests US. Preparing for the Future of HIV/AIDS in Africa: A Shared Responsibility. Washington (DC): National Academies Press (US) Copyright 2011 by the National Academy of Sciences. All rights reserved.; 2011.

18. Deller B, Tripathi V, Stender S, Otolorin E, Johnson P, Carr C. Task shifting in maternal and newborn health care: key components from policy to implementation. Int J Gynaecol Obstet. 2015;130 Suppl 2:S25-31.

19. World Health Organization. Optimizing health worker roles to improve access to key maternal and newborn health interventions through task shifting. Geneva: World Health Organization; 2012.

20. Polus S, Lewin S, Glenton C, Lerberg PM, Rehfuess E, Gulmezoglu AM. Optimizing the delivery of contraceptives in low- and middle-income countries through task shifting: a systematic review of effectiveness and safety. Reprod Health. 2015;12:27.

21. Van Rie A, Patel MR, Nana M, Vanden Driessche $K$, Tabala M, Yotebieng M, et al. Integration and task shifting for TB/HIV care and treatment in highly resource-scarce settings: one size may not fit all. J Acquir Immune Defic Syndr. 2014;65(3):e110-7.

22. Munga MA, Kilima SP, Mutalemwa PP, Kisoka WJ, Malecela MN. Experiences, opportunities and challenges of implementing task shifting in underserved remote settings: the case of Kongwa district, central Tanzania. BMC Int Health Hum Rights. 2012;12:27.

23. Anand TN, Joseph LM, Geetha AV, Chowdhury J, Prabhakaran D, Jeemon P. Task-sharing interventions for cardiovascular risk reduction and lipid outcomes in low- and middle-income countries: A systematic review and meta-analysis. Journal of clinical lipidology. 2018;12(3):626-42.

24. Farley JE. Evaluation of a nurse practitioner-physician task-sharing model for multidrug-resistant tuberculosis in South Africa. 2017;12(8).

25. Kenya Ministry of Health. Task sharing policy guidelines 2017-2030: Expanding access to quality health services through task sharing. Nairobi, Kenya: Kenya Ministry of Health; 2017.

26. East LA, Arudo J, Loefler M, Evans CM. Exploring the potential for advanced nursing practice role development in Kenya: a qualitative study. BMC nursing. 2014;13(1):33.

27. USAID. Assessment phase 2: learn n.d. [Available from: https://assessment-action.net/wpcontent/uploads/2014/03/Phase-2-LEARN-Overview.pdf.

28. United Nations Development Programme. The process of capacity development [cited 2021. Available from: https://www.undp-capacitydevelopment-health.org/en/capacities/capacitydevelopment-process/.

29. Kenya Law. Association of Kenya Medical Laboratory Scientific Officers v Ministry of Health \& another [2019] eKLR 2019 [cited 2019. Available from: http://kenyalaw.org/caselaw/cases/view/174230.

\section{Supplementary Files}


This is a list of supplementary files associated with this preprint. Click to download.

- AppendixABC.docx 